\title{
Clinical significance of plasma cell-free DNA mutations in PIK3CA, AKT1, and ESR1 gene according to treatment lines in ER-positive breast cancer
}

Takashi Takeshita ${ }^{1}$, Yutaka Yamamoto ${ }^{1}$, Mutsuko Yamamoto-lbusuki², Mai Tomiguchi', Aiko Sueta', Keiichi Murakami ${ }^{1}$ and Hirotaka Iwase ${ }^{1 *}$

\begin{abstract}
The somatic activation of PI3K/AKT pathway mutations, PIK3CA and AKT1, and ESR1 mutations in plasma cell-free DNA (cfDNA) has been studied as a non-invasive procedure to quickly assess and monitor disease progression or therapeutic effect in breast cancer (BC) patients, but the clinical significance of these mutations in late treatment lines (TLs) remains unclear. The subjects of this study were a total of 251 plasma samples from 128 estrogen receptor-positive (ER+) BC patients. Of these plasma samples, 133 were from 73 primary BC (PBC) patients, and 118 plasma samples were from 68 metastatic BC (MBC) patients. We developed droplet digital PCR (ddPCR) assays to verify the clinical significance of PIK3CA, AKT1, and ESR1 mutations in these patients. CfDNA PIK3CA mutations were observed in $15.1 \%$ of the PBC patients, while a cfDNA AKT1 mutation was observed in $1.4 \%$ of patients, and cfDNA ESR1 mutations were observed in $2.7 \%$ of patients. Patients with detectable cfDNA PIK3CA mutations were not associated with clinical outcomes. According to the TL, the prevalence of the PIK3CA and ESR1 mutations in cfDNA were lower in early TLs compared with late TLs. In the early TL group, patients with cfDNA PIK3CA mutations had a shorter time to treatment failure (TFF) than patients without mutations $(P=0.035)$. However, there was no statistically significant difference between patients with or without cfDNA ESR1 mutations. However, in the late TL group, patients with cfDNA ESR1 mutations had a shorter TTF than patients without mutations $(P=0.048)$. However, there was no statistically significant difference between patients with or without cfDNA PIK3CA mutations. Since the prevalence of cfDNA AKT1 mutation is low in both PBC and MBC patients, the impact of AKT1 mutations on the prognosis remains unclear. We have demonstrated the difference in the clinical significance of the hotspot PIK3CA, $A K T 1$, and ESR1 mutations in cfDNA for each TL in ER+ BC patients.
\end{abstract}

Keywords: Estrogen receptor-positive breast cancer, Cell-free DNA, PIK3CA mutations, AKT1 mutation, ESR1 mutations

Endocrine therapy (ET) resistance occasionally occurs during the treatment of primary breast cancer $(\mathrm{PBC})$ and inevitably results in metastatic $\mathrm{BC}(\mathrm{MBC})$. Recently, the focused mechanisms of ET resistance include hyperactivation of $P I 3 K / A K T$ pathway. Importantly, somatic activating mutations of PIK3CA and $A K T 1$ affect the

\footnotetext{
* Correspondence: hiwase@kumamoto-u.ac.jp

'Department of Breast and Endocrine surgery, Graduate School of Medical Science, Kumamoto University, 1-1-1 Honjo, Chuo-ku, Kumamoto 860-8556, Japan

Full list of author information is available at the end of the article
}

magnitude of $P I 3 K / A K T$ activation [1]. Meta-analysis of the $\mathrm{BC}$ literature shows that the PIK3CA mutation is present in $20 \%-40 \%$ of all BCs, making this gene the second most frequently mutated in $\mathrm{BC}$, with most mutations being expressed in 'hotspots' in the helical domain (exon (Ex) 9) or the catalytic domain (Ex20) [2]. A somatic mutation in the plekstrin homology domain of AKT1:p.Glu17Lys is found in approximately $4 \%$ of breast tumors [3]. Conversely, recent evidence describing next generation sequencing showed that another key potential mechanism of the failure of ET involves activating 
mutations in the ligand-binding domain of the ESR1 gene [4]. Those mutations cause ligand-independent estrogen receptor (ER) transcriptional activity that does not respond to endocrine manipulation. The estimated frequency from comprehensive studies was $20 \%-50 \%$ for ESR1 mutations in $\mathrm{MBC}$ but that were minimally present in PBC. The potential of using PI $3 K / A K T$ pathway mutations and ESR1 mutations as biomarkers to predict clinical response of ET is currently the focus of many preclinical and clinical studies such as the BOLERO-2 cohort [5]. Additionally, recent digital PCR assays on plasma cell-free DNA (cfDNA) of several cohorts demonstrated the difference in the clinical features between the representative hotspot mutations in $\mathrm{MBC}$, PIK3CA and ESR1 mutations [6-10]. In the BOLERO-2 study, Chandarlapaty and colleagues demonstrated that progression free survival (PFS) benefit of mammalian target of rapamycin (mTOR) inhibitor everolimus was maintained irrespective of PIK3CA mutations, but that was decreased according to the presence of ESR1 mutations $[6,7]$. In another two phase III randomized trials, Fribbens and colleagues reported the effectiveness of the target drug by having the mutations or not. In the SoFEA study, fulvestrant improved PFS of patients with ESR1 mutations compared to exemestane. Meanwhile, in the PALOMA3 study, fulvestrant plus the CDK4/6-inhibitor palbociclib improved PFS regardless of the genomic status of ESR1 or PIK3CA $[8,9]$. We demonstrated the clinical significance of the burden of on-treatment hotspot ESR1 mutations, both in a snapshot and serially in MBC patients in comparison with PIK3CA hotspot mutation status [10]. Although these studies revealed the clinical significance of the target mutations in the early treatment lines (TLs), the clinical significance of PIK3CA, AKT1, and ESR1 mutations in late line treatment in ER+ BC patients is still controversial. In this retrospective study, we demonstrated the clinical significance of the burden of on-treatment hotspot mutations: PIK3CA Ex9:p.Glu542Lys/Val, Glu545Val/Gly/Ala/Gln/ Lys and Gln546Leu/Arg/Pro/Glu/Lys and PIK3CA Ex20:p.His1047Leu/Arg/Tyr and Gly1049Arg/Ser and AKT1:p.Glu17Lys, and ESR1:p.Tyr537Ser/Asn and Asp538Gly in cfDNA in comparison with ER+ PBC patients in each TL of ER+ MBC patients using multiplex droplet digital PCR (ddPCR) assays.

\section{Results and discussion}

A total of 128 patients (251 plasma samples) with breast carcinoma who had an ECOG Performance scale status of 0 or 1 were enrolled in this study. The participants were comprised of 73 women (133 plasma samples) with $\mathrm{PBC}$ and 68 women (118 plasma samples) with MBC. Of these participants, 13 women had plasma samples with both PBC and MBC (Additional file 1: Table S1).
Plasma PIK3CA mutations were found in 15.1\% (11/73) of the PBC patients, the plasma $A K T 1$ mutation was detected in $1.4 \%(1 / 73)$, and the plasma ESR1 mutations were observed in 2.7\% (2/73) (Additional file 1: Table S2). One patient had co-mutations (PIK3CA mutations and $A K T 1$ mutation). This finding agrees with previous studies in which PIK3CA mutations are particularly common, whereas $A K T 1$ and ESR1 mutations occur less frequently in $\mathrm{PBC}$ patients [3-5]. The presence of plasma PIK3CA mutations were statistically associated with invasive lobular carcinoma $(P=0.036)$ and marginally associated with histological grade III carcinoma $(P=$ 0.059) (Additional file 1: Table S3). In the PBC group for recurrence free survival and breast cancer specific survival analyses, the patients were not stratified for the PIK3CA mutation status (Fig. 1a, b and Additional file 1: Tables S4, S5). In general, among the ER+ PBC patients, the PIK3CA mutations had varying clinical outcomes, but they were associated with a favorable prognosis [2]. Due to the difference in the clinical effect of PIK3CA mutations in the helical versus kinase domains, the prognostic effect may not be consistent. This finding suggests that the presence of Ex9 mutations predicted a more favorable outcome, whereas the presence of the Ex20 mutations predicted a relatively poor prognosis [2]. In this study, we could not evaluate the difference in the prognosis for each PIK3CA mutation, since PIK3CA Ex20 mutations were observed in $90.9 \%(10 / 11)$ of patients with PIK3CA mutations, and PIK3CA Ex9 and Ex20 mutations were observed in $10.1 \%(1 / 11)$ of patients with PIK3CA mutations (Additional file 1: Table S2). Interestingly, out of all of the patients, two patients with plasma ESR1 mutations recurred. Investigations on the progression or survival by ESR1 mutations in PBC have not been conducted and are highly desirable. Rudolph and colleagues reported that initial follow-up survival data suggests that the $A K T 1$ :p.Glu17Lys mutant could be associated with increased mortality [3]; however, none of the two patients with plasma $A K T 1$ mutation in this study showed recurrence.

In the total TL analysis, as the TL increased, the frequency of PIK3CA and ESR1 mutations increased due to the accumulation of genetic alterations. PIK3CA and ESR1 mutations were specifically found to be lower in the 4th TL or less (PIK3CA vs ESR1 mutations: $16.7 \%$ vs $25 \%$ in the $1 \mathrm{st} / 2$ nd line and $17 \%$ vs $26 \%$ in the $3 \mathrm{rd} / 4$ th line), compared with those in more than the 5 th TL (PIK3CA vs ESR 1 mutations: $39 \%$ vs $43 \%$ in the 5 th-7th line and $32 \%$ vs $42 \%$ in the 8 th line or more) (Fig. 2a). In the ET line analysis of the MBC patients, PIK3CA, $A K T 1$, and ESR1 mutations were found to be highest in the 4th/5th line (PIK3CA mutations: 39\% (11/28), AKT1 mutation: 7.1\% (2/28), and ESR1 mutations: 61\% (17/ 28)) (Fig. 2b). In the chemotherapy line analysis of the 

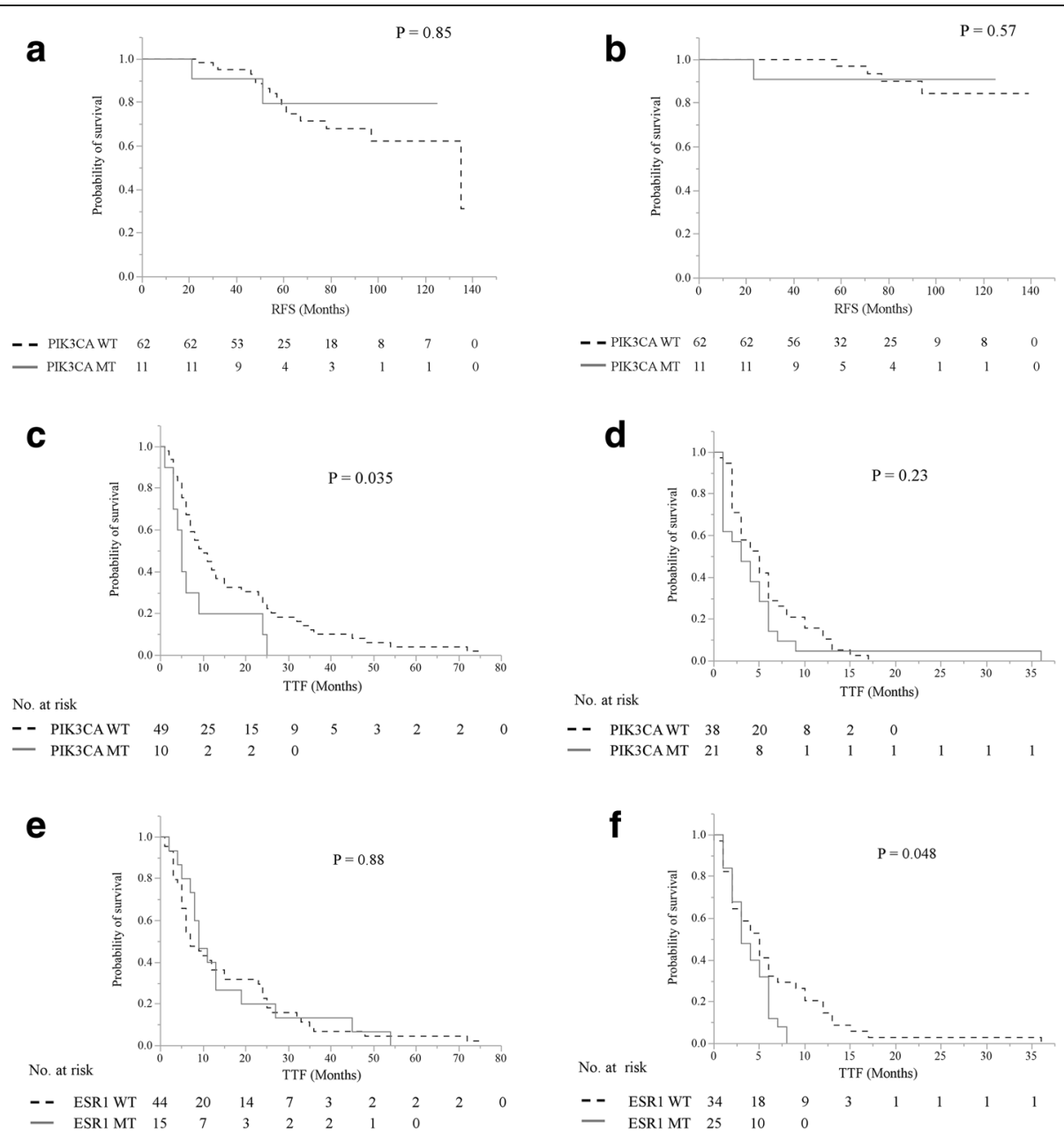

Fig. 1 Kaplan-Meier plots of the association of the presence of CFDNA PIK3CA mutations with RFS; $\mathbf{a}$ and BCSS; $\mathbf{b}$ in the entire cohort and the presence of cfDNA PIK3CA mutations with TTF in less than the 5th line; $\mathbf{c}$ more than the 5th line; $\mathbf{d}$, and the presence of cfDNA ESR1 mutations with TTF in less than the 5th line; e and more than the 5th line; $\mathbf{f}$ in the entire cohort. The presence of cfDNA PIK3CA mutations and cfDNA ESR1 mutations were defined as either positive or negative. Abbreviations: cfDNA, cell-free DNA; RFS, relapse-free survival; BCSS, breast cancer-specific survival; TTF, time to treatment failure

MBC patients, PIK3CA and ESR1 mutations were found to be highest in the 6th line or more (PIK3CA mutations: 36\% (4/11) and ESR1 mutations: 64\% (7/11)) (Fig. $2 \mathrm{c})$. To the best of our knowledge, this result that PIK3CA and ESR 1 mutations were more frequent in the late TL is a new finding. Of these mutations, ESR1 mutations were slightly more frequent than PIK3CA mutations. Meanwhile, plasma $A K T 1$ mutation was rare in $\mathrm{MBC}$ and it did not correlate with the TL $(3.6 \%$ in the 5th-7th line and $3.2 \%$ in the 8th line or more) (Fig. 2). $A K T 1$ mutation coexisted with PIK3CA mutations in one MBC patient.

Next, we analyzed whether PIK3CA and ESR1 mutations detected in cfDNA were associated with the differential benefit of time to treatment failure (TTF) of the early or late TL (Fig. 1c-f). By examining the difference in the TTF of the early or late TL with cfDNA PIK3CA and ESR1 mutations, we could create subgroups of patients whose TL was either less than or more than the 5th line, since patients in less than the 5th line had a longer TTF than patients in more than the 5th line $(P<$ 0.0001 ) (Additional file 1: Figure S1). Since the prevalence of the $A K T 1$ mutation is low in both $\mathrm{PBC}$ and MBC patients, the impact of $A K T 1$ mutation on prognosis remains unclear. Our data indicated that patients with cfDNA PIK3CA mutations had a shorter TTF than patients without mutations in the early TL group $(P=$ $0.035)$, but there were no statistically significant differences in the patients with or without cfDNA PIK3CA mutations $(P=0.23)$ in the late TL group (Fig. 1c, d). Paradoxically, as the TL advanced, there was a tendency for PIK3CA Ex9 mutations, a more favorable mutation, to increase. PIK3CA Ex20 mutations, a more aggressive mutation, were expressed stably over the course of 


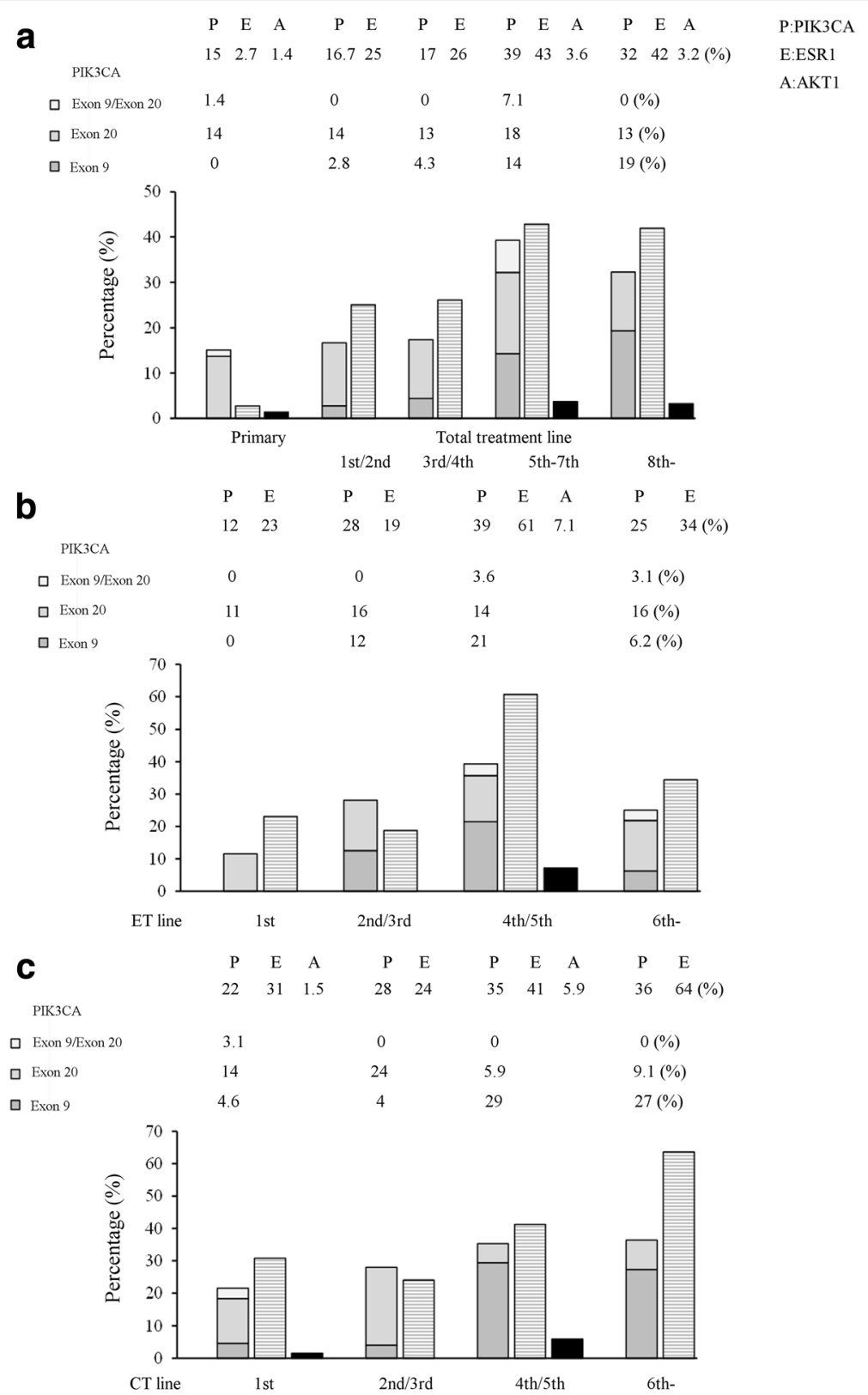

Fig. 2 Distributions of PIK3CA, AKT1, and ESR1 mutations according to each TL in ER-positive breast cancer. The bar graphs show PIK3CA mutations (gray monochrome), ESR1 mutations (gray horizontal stripes), and AKT1 mutation (black monochrome) from the left in each category. a The subgroup in the primary and each TL (the 1st/2nd line, the 3rd/4th line, the 5th-7th line, and the 8th line or more line) were omitted. The subgroup in each endocrine therapy line; $\mathbf{b}$, and in each chemotherapy line; $\mathbf{c}$, (the 1 st line, the 2nd/3rd line, the 4th/5th line, and the 6th line or more line) were omitted. Abbreviations: ER, estrogen receptor; P, PIK3CA mutations; E, ESR1 mutations; A, AKT1 mutation

treatment (Fig. 2). Meanwhile, there was no statistically significant differences in patients with cfDNA ESR1 mutations or not in the early TL group $(P=0.88)$, but patients with cfDNA ESR1 mutations had a shorter TTF than patients without mutations in the late TL group ( $P$ $=0.048)$ (Fig. 1e, f). Additionally, as the TL advanced, there was a tendency for patients treated with ET to decrease $(83 \%$ in the 1 st $/ 2$ nd line, $74 \%$ in the $3 \mathrm{rd} / 4$ th line, $64 \%$ in the 5 th -7 th line, and $68 \%$ in the 8 th line or more) (Additional file 1: Figure S2A) and there was a tendency for the best overall response for the treatment to be worse (the frequency of progressive disease: $33 \%$ in the $1 \mathrm{st} / 2$ nd line, $30 \%$ in the $3 \mathrm{rd} / 4$ th line, $50 \%$ in the 5th-7th line, and $45 \%$ in the 8th line or more) (Additional file 1: Figure S2B). These results suggested the presence of plasma ESR1 mutations, which may be relevant for the choice of treatment of the ER+ MBC patients even in the higher TLs. 


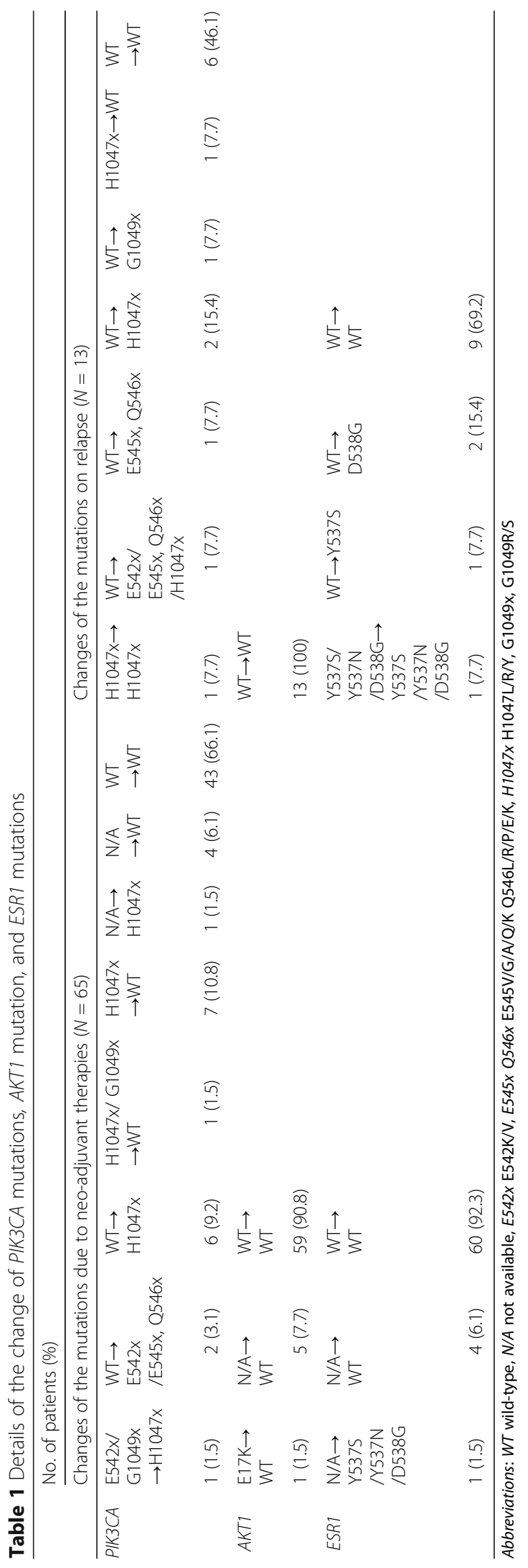


Recently, potential interest in the mutations accompanying disease progression or treatment has been increasing, since they may be a clue for the further treatment. Our data indicated that of the $65 \mathrm{PBC}$ patients treated with neoadjuvant therapy, 9 patients (13.8\%) acquired or maintained PIK3CA mutations, but 8 patients (12.3\%) lost PIK3CA mutations after neoadjuvant therapy. Meanwhile, one patient (1.5\%) lost AKT1 mutation and one patient (1.5\%) acquired ESR1 mutations after neo-adjuvant therapy. Of the $13 \mathrm{BC}$ patients with both primary and recurrence blood samples, 6 patients (46.2\%) acquired or maintained PIK3CA mutations, but 1 patient (7.7\%) lost PIK3CA mutations during relapse. Meanwhile, 4 patients $(30.8 \%)$ acquired or maintained ESR1 mutations during relapse (Table 1). These results were very interesting, but we could not determine their clinical relevance due to the small sample size.

\section{Conclusions}

Our study demonstrates the difference in the clinical significance of PIK3CA, AKT1, and ESR1 hotspot mutations for each TL in ER+ BC patients using multiplex ddPCR assays. To the best of our knowledge, limited data exist on whether the detection of these mutations may be useful as a biomarker for predicting the effect of late line treatment.

\section{Additional file}

Additional file 1: Methods and Supplementary information. (DOCX $407 \mathrm{~kb}$ )

\section{Abbreviations}

cfDNA: cell-free DNA; ddPCR: droplet dPCR; ER: Estrogen receptor; ET: Endocrine therapy; Ex: Exon; MBC: Metastatic BC; PBC: Primary breast cancer; PFS: Progression free survival; TL: Treatment line; TTF: Time to treatment failure

\section{Acknowledgements}

Not applicable

\section{Funding}

This work was supported in part by a grant-in-aid (project numbers 17 K16511 and 15 K10057) for scientific research from the Ministry of Education, Science and Culture of Japan.

\section{Availability of data and materials}

Please contact the corresponding author for all data requests.

\section{Authors' contributions}

The study was conceived by $\Pi$ and $H I$. $\Pi$ performed ddPCR assay, generated and assembled the data. TT, YY, MY-I, MT, AS, KM, and HI developed and performed the data analysis and wrote the manuscript. All authors have read and approved of the final version of this manuscript.

\section{Ethics approval and consent to participate}

The Ethics Committee of Kumamoto University Graduate School of Medicine (Kumamoto, Japan) approved the study protocol (\#1857). Informed consent was obtained from all individual participants included in the study.

\section{Competing interests}

All of the authors declare that they have no actual, potential, or perceived conflict of interest regarding the manuscript submitted for review.

\section{Publisher's Note}

Springer Nature remains neutral with regard to jurisdictional claims in published maps and institutional affiliations.

\section{Author details}

${ }^{1}$ Department of Breast and Endocrine surgery, Graduate School of Medical Science, Kumamoto University, 1-1-1 Honjo, Chuo-ku, Kumamoto 860-8556, Japan. ${ }^{2}$ Department of Molecular-Targeting Therapy for Breast Cancer, Kumamoto University Hospital, 1-1-1 Honjo, Chuo-ku, Kumamoto 860-8556, Japan.

Received: 28 November 2017 Accepted: 5 February 2018

Published online: 26 February 2018

\section{References}

1. Loi S, Haibe-Kains B, Majjaj S, Lallemand F, Durbecq V, Larsimont D, Gonzalez-Angulo AM, Pusztai L, Symmans WF, Bardelli A, et al. PIK3CA mutations associated with gene signature of low mTORC1 signaling and better outcomes in estrogen receptor-positive breast cancer. Proc Natl Acad Sci U S A. 2010:107:10208-13.

2. Dirican E, Akkiprik M, Ozer A. Mutation distributions and clinical correlations of PIK3CA gene mutations in breast cancer. Tumour Biol. 2016:37:7033-45.

3. Rudolph M, Anzeneder T, Schulz A, Beckmann G, Byrne AT, Jeffers M, Pena C, Politz O, Kochert K, Vonk R, Reischl J. AKT1 (E17K) mutation profiling in breast cancer: prevalence, concurrent oncogenic alterations, and bloodbased detection. BMC Cancer. 2016;16:622.

4. Angus L, Beije N, Jager A, Martens JW, Sleijfer S. ESR1 mutations: moving towards guiding treatment decision-making in metastatic breast cancer patients. Cancer Treat Rev. 2017;52:33-40.

5. Hortobagyi GN, Chen D, Piccart M, Rugo HS, Burris HA, 3rd, Pritchard KI, Campone M, Noguchi S, Perez AT, Deleu I, et al: Correlative analysis of genetic alterations and Everolimus benefit in hormone receptor-positive, human epidermal growth factor receptor 2-negative advanced breast cancer: results from BOLERO-2. J Clin Oncol 2016, 34:419-426.

6. Moynahan ME, Chen D, He W, Sung P, Samoila A, You D, Bhatt T, Patel P, Ringeisen F, Hortobagyi GN, et al. Correlation between PIK3CA mutations in cell-free DNA and everolimus efficacy in HR+, HER2- advanced breast cancer: results from BOLERO-2. Br J Cancer. 2017;116:726-30.

7. Chandarlapaty S, Chen D, He W, Sung P, Samoila A, You D, Bhatt T, Patel P, Voi M, Gnant $M$, et al. Prevalence of ESR1 mutations in cell-free DNA and outcomes in metastatic breast cancer: a secondary analysis of the BOLERO-2 clinical trial. JAMA Oncol. 2016:2:1310-5.

8. Cristofanilli M, Turner NC, Bondarenko I, Ro J, Im SA, Masuda N, Colleoni M, DeMichele A, Loi S, Verma S, et al. Fulvestrant plus palbociclib versus fulvestrant plus placebo for treatment of hormone-receptor-positive, HER2negative metastatic breast cancer that progressed on previous endocrine therapy (PALOMA-3): final analysis of the multicentre, double-blind, phase 3 randomised controlled trial. Lancet Oncol. 2016;17:425-39.

9. Fribbens C, O'Leary B, Kilburn L, Hrebien S, Garcia-Murillas I, Beaney M, Cristofanilli M, Andre F, Loi S, Loibl S, et al. Plasma ESR1 mutations and the treatment of estrogen receptor-positive advanced breast cancer. J Clin Oncol. 2016;34:2961-8

10. Takeshita T, Yamamoto Y, Yamamoto-lbusuki M, Tomiguchi M, Sueta A, Murakami K, Omoto Y, Iwase H. Analysis of ESR1 and PIK3CA mutations in plasma cell-free DNA from ER-positive breast cancer patients. Oncotarget. 2017:8:52142-55. 